\title{
L'École de pharmacie de l’Université de Coimbra (1902-1911)
}

\author{
par João Rui Pita, Victoria Bell, Ana Leonor Pereira*
}

\section{Introduction}

À la fin du XIX $X^{\mathrm{e}}$ siècle et au début du $\mathrm{XX}^{\mathrm{e}}$ siècle, l'enseignement pharmaceutique était un sujet très important pour les pharmaciens portugais. C'était aussi une question majeure dans beaucoup de pays européens.

En 1836, les Écoles de pharmacie de Lisbonne, Porto et Coimbra ont été créées. C'était la première fois qu'on créait des Écoles de pharmacie au Portugal. À l'Université de Coimbra, il existait cependant un enseignement pharmaceutique depuis le $\mathrm{XVI}^{\mathrm{e}}$ siècle. C'était un enseignement pratique qui était réalisé dans les pharmacies d'officine, sous la direction d'un apothicaire. Un enseignement avait lieu à l'Université et les étudiants y passaient leurs examens pour l'obtention du diplôme. Depuis 1772, l'enseignement pharmaceutique à l'Université de Coimbra était dispensé au Laboratório Químico (Laboratoire chimique) et au Dispensatório Farmacêutico (Dispensaire pharmaceutique, qui était la pharmacie de l'hôpital de l'Université de Coimbra). Les études de pharmacie se déroulaient sur quatre années : deux années de pratique au Laboratório Químico et deux années au Dispensatório Farmacêutico. Le Laboratoire de chimie était un ardent défenseur des doctrines chimiques modernes de Lavoisier, surtout en raison des travaux de Vicente Seabra (1764-1804) et de Tomé Rodrigues Sobral (17591829). En 1772, l'enseignement pharmaceutique bénéficia d'un lieu spécialement adapté au sein des installations universitaires. Il est important de noter qu'à partir de 1772, l'enseignement pharmaceutique au Portugal commença à donner à ses élèves une formation en chimie, selon les théories les plus récentes en pharmacie. Antoine Baumé a été traduit et adapté par le professeur de matière médicale et

\footnotetext{
* $\quad$ Faculté de Pharmacie, CEIS20, Université de Coimbra, Pôle des Sciences de la Santé, Azinhaga de Santa Comba, 3000-548 Coimbra (Portugal) (Portugal)

Faculté des Lettres, CEIS20, Université de Coimbra, Largo da Porta Férrea, 3004-530 Coimbra
} 
de pharmacie de la Faculté de médecine José Francisco Leal, qui était le directeur du Dispensaire pharmaceutique. À Lisbonne et Porto, en revanche, il n'y avait pas enseignement pharmaceutique.

Les Écoles de pharmacie de Coimbra, Lisbonne et Porto, fondées en 1836 sous l'impulsion du ministre Passos Manuel (1801-1862), l'une des figures les plus importantes du libéralisme au Portugal, ont été créées avec un programme d'étude adapté spécifiquement à la formation des pharmaciens : enseignement théorique en zoologie, botanique, physique, minéralogie, etc. ; et enseignement théorique et pratique en chimie et technique pharmaceutique. À Coimbra, l'enseignement était un peu différent de celui délivré à Lisbonne et à Porto : le point de départ était l'enseignement délivré au Dispensatório Farmacêutico.

\section{L'enseignement pharmaceutique au Portugal (première moitié du $\mathrm{XX}^{\mathrm{e}}$ siècle)}

Le contenu de la réforme des études de 1836 a perduré jusqu'en 1902, date à laquelle l'enseignement pharmaceutique fut considéré comme faisant partie de l'enseignement supérieur.

En 1902 (loi du 19 juillet et règlement du 27 novembre), l'enseignement de la pharmacie au Portugal fut réorganisé dans les trois Écoles de pharmacie du pays. La loi a établi l'impôt sur les spécialités pharmaceutiques, objet de controverses qui servait à financer l'enseignement pharmaceutique.

Par le décret du 26 mai 1911 et le règlement du 18 août de la même année, l'enseignement de la pharmacie fut revu et devint autonome par rapport aux Facultés de médecine et à l'enseignement médical. L'École de pharmacie de l'Université de Coimbra ouvrit ses propres locaux universitaires pour la première fois en 1915. En 1918, le décret $\mathrm{n}^{\circ} 4.653$ a réorganisé une nouvelle fois l'enseignement pharmaceutique dans les trois Écoles du pays, à travers une nouvelle réforme des études. Les écoles prirent le nom d'Écoles supérieures de pharmacie (Escolas Superiores de Farmácia). En 1919 (décret $\mathrm{n}^{\circ}$ 5.463), les Écoles de pharmacie ont commencé à délivrer un diplôme au niveau des autres diplômes universitaires. En 1921, les Écoles de pharmacie de Lisbonne, Porto et Coimbra (décrets $n^{\circ} 7.238$ et $n^{\circ} 7.355$ pour Porto, $n^{\circ} 7.668$ pour Coimbra et $\mathrm{n}^{\circ} 7.700$ pour Lisbonne) ont acquis le statut de Facultés.

L'enseignement pharmaceutique fut à nouveau réformé en 1926 (décret $n^{\circ}$ 12.698). En 1928, la Faculté de pharmacie de Coimbra cessa d'exister (décret $\mathrm{n}^{\circ} 15.365$ ). Les Facultés de pharmacie ont été réorganisés en 1930 (décret $n^{\circ} 18.432$ ) et en 1932 (décret $n^{\circ} 21.853$ ). L'enseignement de la pharmacie fut rénové : on créa des Écoles de pharmacie à l'Université de Coimbra et à l'Université de Lisbonne ; la Faculté de pharmacie de Porto fut maintenue. 
Ces transformations étaient le résultat de la politique du régime intitulée « Estado Novo » (État Nouveau). Avec cette nouvelle politique, dans les années 1930 à 1970, beaucoup de mesures législatives normalisatrices de la pharmacie et de l'enseignement pharmaceutique au Portugal ont été promulguées. En 1968, les Facultés de pharmacie de Lisbonne et de Coimbra furent rétablies.

\section{L'enseignement pharmaceutique a l'Ecole de Pharmacie de Coimbra (1902)}

\section{- La réforme de 1902}

En 1902, pour la première fois au Portugal, l'enseignement pharmaceutique fut considéré comme un enseignement supérieur et, pour la première fois également, le cursus fut organisé sur deux ans, avec enseignement de quatre disciplines (deux disciplines par an), se rapprochant ainsi du programme d'études des autres cursus universitaires. En 1902, l'Université de Coimbra était la seule université au Portugal. Les universités de Lisbonne et Porto furent en effet fondées seulement en 1911, après la révolution républicaine de 1910.

Cette réforme de 1902 ne se préoccupait pas seulement du contenu des études. C'était aussi une réforme de l'enseignement et une réforme de l'organisation des Écoles de pharmacie qui s'appliquera dès lors à Lisbonne, Porto et Coimbra.

Bien que l'enseignement pharmaceutique ait continué à être dépendant de la Faculté de médecine de Coimbra et des Écoles médicales et chirurgicales de Lisbonne et Porto, cette réforme du contenu des études mit fin à la double voie d'accès à la profession, établie par la réforme de Passos Manuel en 1836. Dans le programme des études de 1836, ceux qui souhaitaient être pharmaciens pouvaient en effet suivre une des deux voies suivantes : a) s'inscrire à une des trois Écoles de pharmacie du pays et pratiquer la pharmacie dans une pharmacie d'officine, sous la direction du pharmacien propriétaire, et terminer par un examen à l'École de pharmacie ; b) s'inscrire à une des Écoles de pharmacie et suivre le programme défini pour ces études. Dans le premier cas, c'étaient des pharmaciens de $2^{\mathrm{e}}$ classe. Les étudiants suivant le second cursus étaient pharmaciens de $1^{\text {re }}$ classe. Toutefois, les deux voies d'accès à la profession permettaient de pratiquer exactement le même type d'exercice professionnel. Il y avait, à ce propos, beaucoup de controverses et de désaccords entre pharmaciens portugais.

La réforme de 1902 a uniformisé l'enseignement de la pharmacie : tous ceux qui souhaitaient devenir pharmaciens devaient suivre le régime d'études des Ecoles de pharmacie. Les législateurs ont pris en compte ce qui se passait à l'étranger, comme par exemple en France, en Allemagne, en Italie ou en Espagne. 


\section{- L'admission à l'École de pharmacie}

La réforme de 1902 a introduit des nouveautés au niveau de l'admission aux études de pharmacie, bien qu'elle n'ait pas établi une voie d'accès unique. Il y avait en effet plusieurs possibilités d'accès aux Écoles de pharmacie : de la pratique professionnelle au cours complémentaire des lycées, en passant par des habilitations intermédiaires, comme par exemple, le cours général des lycées et trois années de pratique pharmaceutique. Il fut ainsi établi un régime transitoire qui permettait aux professionnels pharmaciens ayant un diplôme obtenu à travers l'ancien régime des études de suivre le nouveau cursus de pharmacie.

\section{- L'organisation des cours}

Selon la réforme de 1902, la formation de pharmaciens était orientée selon de nouvelles valeurs : a) rapprochement de son organisation à d'autres cursus d'études supérieures, notamment le cursus de médecine ; b) valorisation institutionnelle du cursus, investissement dans la qualification du personnel enseignant (professeurs titulaires de chaire et professeurs remplaçants), préparateurs et administratifs ; c) organisation des organes de l'École (direction et Conseil scolaire), nouvelles méthodes d'enseignement avec des cours théoriques, démonstrations expérimentales et travaux pratiques ; d) évaluation écrite et orale, examens par disciplines et examen général.

La réforme de 1902 établissait encore : le modèle de concours pour les enseignants et autres techniciens des Écoles de pharmacie ; les critères auxquels devaient obéir les installations ; l'organisation de la bibliothèque ; l'impôt sur les spécialités pharmaceutiques. Cet impôt consistait en l'application d'une taxe sur les spécialités pharmaceutiques (nationales et étrangères) et sur les eaux minérales et médicinales. Les sommes ainsi obtenues étaient destinées à financer l'enseignement pharmaceutique, ce qui causa une énorme controverse au sein du monde pharmaceutique.

La communauté pharmaceutique a considéré la réforme de 1902 comme une réforme acceptable, sans être idéale. La réforme de 1902 fut comprise comme un premier pas vers l'amélioration du cursus de pharmacie et sa mise à niveau par rapport aux autres cursus d'études supérieures. Pour les pouvoirs publics, la réforme de l'enseignement pharmaceutique de 1902 entraînait une augmentation significative de la qualité de la formation des pharmaciens portugais.

\section{- Le programme d'études}

Le programme d'études établi par la réforme 1902 fut structuré de la façon suivante :

- $1^{\text {re }}$ année : $1^{\text {re }}$ discipline : Histoire naturelle des drogues. Posologie. Pratique de laboratoire; $2^{\mathrm{e}}$ discipline : Pharmacie chimique, Analyses micro- 
scopiques et chimiques appliquées à la médecine et à la pharmacie. Pratique de laboratoire ;

- $2^{\mathrm{e}}$ année $: 3^{\mathrm{e}}$ discipline : Pharmacotechnie, Stérilisations et Pratique de laboratoire pharmaceutique ; $4^{\mathrm{e}}$ discipline : Analyses toxicologiques, Chimie médicolégale, Modifications et falsifications des médicaments et des aliments. Pratique de laboratoire de chimie.

La réforme de 1902 prévoyait aussi un « Cours auxiliaire de déontologie et législation pharmaceutique », facultatif, qui devait être enseigné par un professeur remplaçant ou suppléant. Ce cours commença en 1906-1907 avec le professeur Victor Henriques Aires Móra. À partir de 1911, ce cours devint obligatoire.

En analysant les disciplines, nous avons pu voir que la chimie analytique appliquée à la santé publique avait été mise en valeur. Rappelons que la falsification des aliments et des médicaments, le problème des empoisonnements, les analyses de produits toxiques, les analyses et la qualité des eaux, étaient des préoccupations majeures pour la santé publique. En fournissant aux pharmaciens un ensemble de savoirs scientifiques afin qu'ils puissent travailler dans ces domaines, l'État attribuait à ce groupe professionnel des compétences qui se reflétaient dans leurs pratiques en matière de la santé publique.

À l'occasion de la réforme de 1902, on a renforçé la dimension pharmaceutique relative au médicament, de l'étude des drogues jusqu'à la maîtrise de la technique pharmaceutique. On signalera également que l'enseignement de la stérilisation, une matière en rapport avec la microbiologie (bien que l'enseignement de la bactériologie ne soit pas une discipline autonome) fut réalisé dans le souci de se rapprocher des besoins de l'industrie pharmaceutique, mais de façon limitée. Ce n'est qu'à l'occasion des réformes ultérieures que l'on va prêter davantage d'attention à ce sujet.

Le «Cours auxiliaire de déontologie et législation pharmaceutique » s'imposait en raison des exigences législatives et normalisatrices qui se faisaient sentir dans l'exercice de la profession, mais aussi comme outil déontologique et juridique pour le pharmacien portugais.

\section{- Les professeurs de l'École de pharmacie}

Le corps enseignant de l'École de pharmacie durant la réforme de l'enseignement de 1902 était le suivant :

- 1903-1904 : $1^{\text {re }}$ discipline : Poste vacant : Lúcio Martins da Rocha, professeur de la Faculté de médecine de l'Université de Coimbra, de matière médicale et pharmacie ; $2^{\mathrm{e}}$ discipline : Poste vacant : Francisco José de Sousa Gomes, professeur de la Faculté de philosophie (ce professeur a parfois été remplacé par Álvaro José da Silva Basto, professeur de la Faculté de philosophie) ; $3^{\mathrm{e}}$ discipline : Vicente José de Seiça ; $4^{\mathrm{e}}$ discipline : Joaquim dos Santos e Silva. Professeur remplaçant : vacant. 


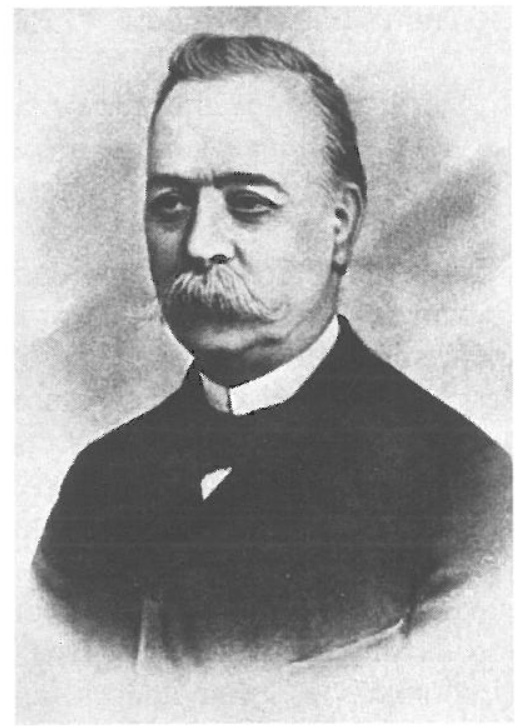

Le professeur Joaquim dos Santos e Silva (in Noticias Farmacêuticas, 4, 1937-1938).

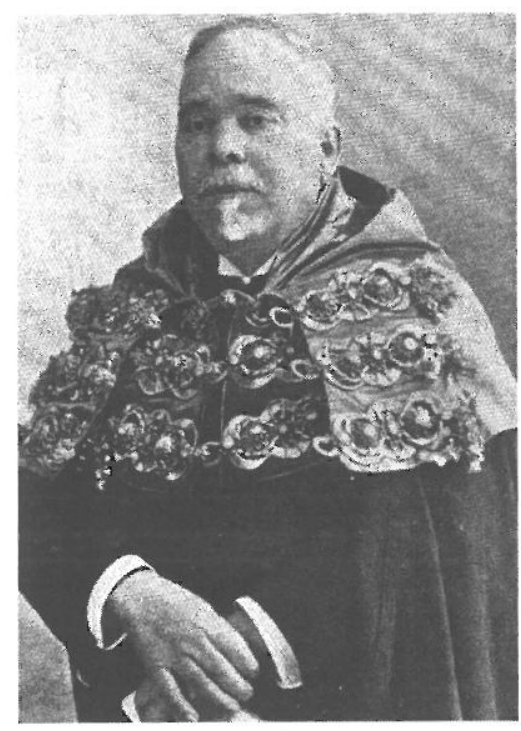

Le professeur Manuel José Fernandes Costa (in Noticias Farmacêuticas, 4, 1937-1938).

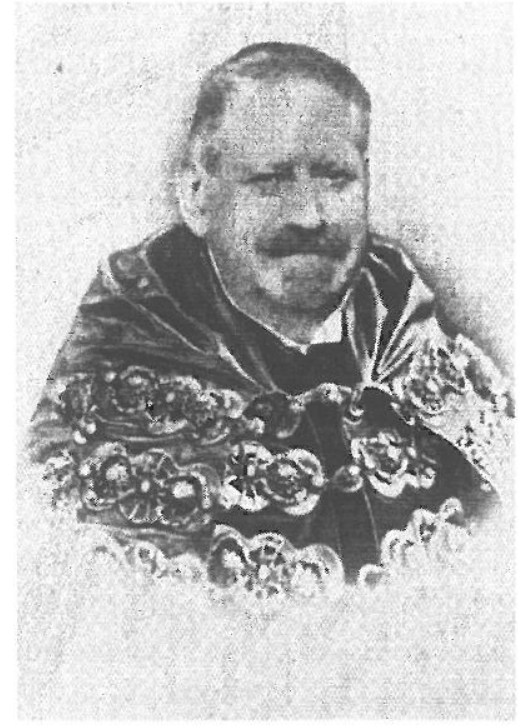

Le professeur Victor Henriques Aires Mora, le premier professeur de Déontologie et Législation (in Notícias Farmacêuticas, 4, 1937-1938).

- 1904-1905 à 1906-1907: $1^{\text {re }}$ discipline : Manuel José Fernandes Costa ; $2^{\mathrm{e}}$ discipline : José Cypriano Rodrigues Diniz ; $3^{\mathrm{e}}$ discipline : Vicente J. Seiça ; $4^{\mathrm{e}}$ discipline: J. Santos e Silva, Professeur remplaçant : Victor Henriques Aires Mora.

- 1907-1908 à 1910-191 : $1^{\text {re }}$ discipline : Manuel J.F. Costa ; $2^{\mathrm{e}}$ discipline : José C.R. Diniz; $3^{\mathrm{e}}$ discipline : Vicente J. Seiça ; $4^{\mathrm{e}}$ discipline : Francisco J.S. Gomes (ce professeur a parfois été remplacé par Álvaro J.S. Basto) ; Professeur remplaçant : Victor H.A. Mora.

La formation de base des professeurs était surtout la pharmacie et la médecine. Certains avaient essentiellement des préoccupations d'enseignement, leurs activités scientifiques n'étant pas significatives ; d'autres développaient égale- 
ment une activité scientifique à travers des publications. Lúcio Rocha (18641950) était médecin et professeur de la Faculté de médecine de l'Université de Coimbra de matière médicale. José Cypriano Diniz (1876-1954) était médecin. Sousa Gomes (1860-1911) et Álvaro Basto (1873-1924) étaient chimistes. Vicente Seiça (1858-1928), Santos e Silva (1842-1906) et Fernandes Costa (1870-1952) étaient pharmaciens. Victor Mora (1861-1933) était médecin et pharmacien.

\section{- La recherche}

L'École de pharmacie de l'Université de Coimbra avait, entre 1902 et 1911, des ressources financières limitées. Entre 1902 et 1915, elle ne disposait pas de ses propres locaux. C'est seulement en 1915 que l'Université de Coimbra lui attribua un bâtiment. La recherche, par conséquent, était jusqu'à cette date peu développée. Le principal objectif entre 1902 et 1911 fut la consolidation de l'École, l'obtention de locaux spécifiques, l'assurance d'avoir des professeurs, d'augmenter le nombre d'étudiants et de fournir à ces derniers la meilleure éducation pharmaceutique possible.

Parmi ceux qui ont réalisé une œuvre scientifique, on peut citer Joaquim dos Santos e Silva, pharmacien qui a acquis une renommée dans le domaine des analyses chimiques appliquées à la santé publique, en particulier celles relatives aux eaux et aliments. Il publia le premier traité de chimie analytique du Portugal, Elementos de analyse chimica qualitativa (Éléments d'analyse chimique qualitative, $1^{\text {re }}$ édition $1874 ; 2^{\mathrm{e}}$ éd. $1883 ; 3^{\mathrm{e}}$ éd. 1891). Il fit également des analyses de plantes médicinales et a publié des articles à ce sujet. Santos e Silva a travaillé à l'Université de Coimbra avec Bernhard Tollens (1841-1918). Puis, il a eu l'occasion d'effectuer un stage en Allemagne (Göttingen) auprès de Bernhard Tollens, Friedrich Wöhler (1800-1882) et Hans Hübner (1837-1884), puis à Bonn auprès de August Kekulé (1829-1896) et Otto Wallach (1847-1931). Ce qu'il a appris a beaucoup influencé sa vision scientifique et ses publications. Il a publié aussi un petit livre, Factoren-tabellen zur ausführung chemischer rechnungen mittels der Von L. Meyer und K. Seubert Gegebenen atomgewichte (1887).

Par ailleurs, Vicente Seiça, un autre pharmacien, a été membre de plusieurs sociétés scientifiques au Portugal et à l'étranger. Il était membre de la Société chimique de Paris. Il est l'auteur de plusieurs articles originaux publiés dans quelques revues portugaises comme O Instituto, Coimbra Médica, Gazeta de Pharmacia, Pharmacia Portugueza, Jornal da Sociedade Pharmaceutica Lusitana, etc. Ses travaux scientifiques et techniques ont surtout porté sur la technique pharmaceutique, la pharmacotechnie, la législation pharmaceutique et l'enseignement pharmaceutique. Manuel José Fernandes Costa et José Cypriano Rodrigues Diniz, quant à eux, ont surtout publié après 1911. Fernandes Costa a 
principalement publié des travaux de pharmacognosie, et Diniz quelques travaux de revue générale sur la chimie, l'enseignement pharmaceutique et la pharmacie. Manuel José Fernandes Costa est l'un des professeurs les plus importants de l'histoire de la Faculté de pharmacie de Coimbra.

On peut également citer les travaux qui ont été présentés pour le concours à l'École de pharmacie en 1904. Ce sont des travaux de recherche surtout sur des thèmes de chimie pharmaceutique et de technique pharmaceutique : António Carvalho da Fonseca, Vinhos medicinaes ; Benjamim Gonçalves Craveiro, Algumas considerações sobre a pharmacia portugueza ; Joaquim de Jesus Cardoso e Sousa, Valor hygienico e processos actuaes de analyse das aguas potaveis ; José Collaço Alves Sobral, O phosphoro e seus principaes compostos ; José Cypriano Rodrigues Diniz, Solanaceas medicamentosas portuguesas. Meimendros (Hyosciamus niger L., Hyosciamus albus L.) ; Luiz d'Almeida, A pharmacia e o exercicio profissional (a traços rapidos) ; Manuel José Fernandes Costa, Hypericum androsoemum L. ; Victor Ayres Mora, O chá. Sua composição química, preparação e falsificação. Certains de ces travaux sont des travaux de recherche en laboratoire.

En conclusion, l'objectif principal de l'École de pharmacie était surtout la formation de pharmaciens et la consolidation de son existence au sein des facul-

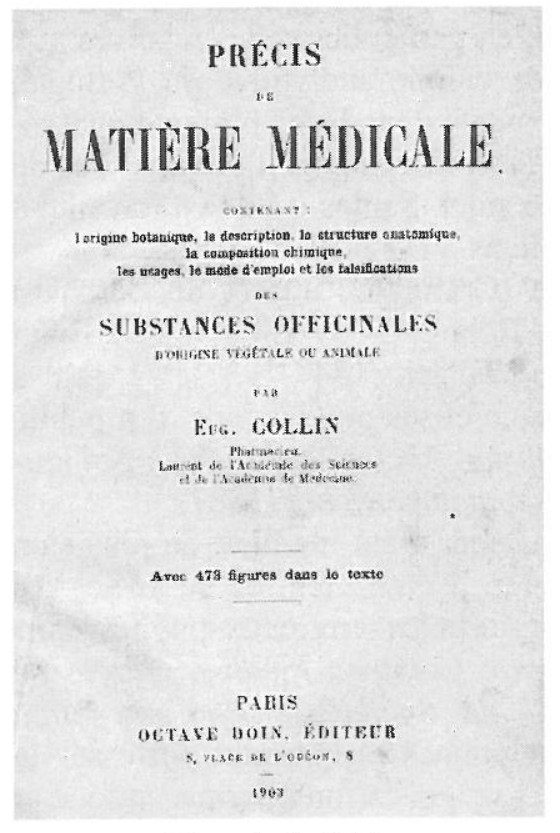

Livre de E. Collin, Précis de matière médicale (1903). tés de l'Université de Coimbra. Les professeurs ont par ailleurs rendu possible l'introduction de la recherche.

\section{-Les manuels d'enseignementetl'influence} française

La littérature scientifique proposée aux étudiants était, en grande partie, d'origine étrangère. Nous n'avons trouvé que trois ouvrages portugais : la Pharmacopêa Portugueza (Pharmacopée portugaise ; la troisième pharmacopée officielle portugaise, 1876) ; l'ouvrage de Júlio Sacadura Botte, médecin professeur de la Faculté de médecine, Pharmacia. Elementos de Pharmacotechnia (Pharmacie. Éléments de Pharmacotechnie) ; et celui de Joaquim dos Santos e Silva, Elementos de analyse chimica qualitativa (Éléments d'analyse chimique qualitative).

Tous les autres ouvrages recommandés étaient étrangers et tous étaient édités en langue 
française. Les manuels étrangers étaient les suivants : E. Collin, Précis de matière médicale ; Crollas, Moreau, Pharmacie chimique ; L. Prunier, Médicaments chimiques ; Edmond Dupuy, Cours de pharmacie ( $2^{\mathrm{e}}$ éd.) ; J. Tarbouriech, Technique des analyses chimiques; Fonzes-Diacon, Précis de toxicologie.

L'usage d'ouvrages étrangers démontre une certaine insuffisance dans la production scientifique portugaise, mais c'était cependant une pratique universitaire courante. Pour les étudiants qui avaient connu la réforme de 1902, l'étude de ces manuels, quoique en langue française, était une condition essentielle pour leur admission.

\section{- Les étudiants}

Entre 1902 et 1911, 105 étudiants furent enregistrés sur le livre d'inscriptions du cursus de pharmacie de l'École de pharmacie de l'Université de Coimbra.

Il existait une grande variété d'origine scolaire des étudiants en pharmacie, surtout pour les pharmaciens diplômés selon le régime d'études de 1836 et les étudiants de l'ancien régime inscrits pour être habilités par le nouveau régime d'études. En moyenne, il y avait douze étudiants par année scolaire : un nombre réduit comparé à celui d'autres facultés de l'Université de Coimbra. Toutefois, dans le livre des examens finaux, on retrouve 257 enregistrements, non seulement pour les examens d'étudiants réguliers mais aussi de tous les autres du régime transitoire stipulé par la réforme de 1902 . Les étudiants examinés étaient pour la plupart du sexe masculin (il n'y avait que sept femmes).

À l'époque, l'École de pharmacie essayait de s'affirmer dans le panorama universitaire portugais. Mais les études pharmaceutiques étaient un atout important pour la professionnalisa-

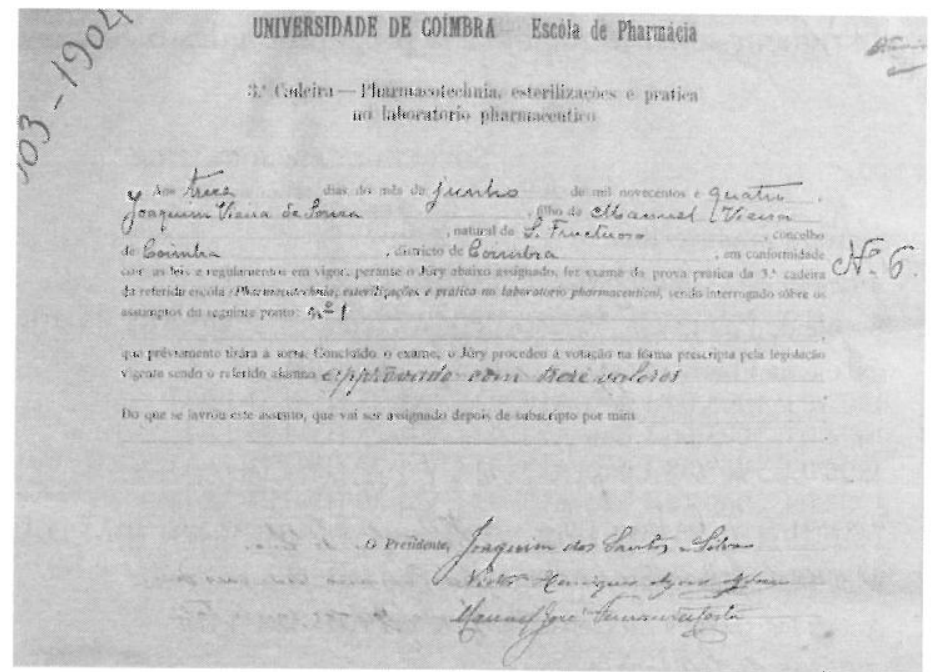

Enregistrement de la classification d'un élève à la $3^{\text {a }}$ Cadeira Farmacotecnia, esterilizações e prática no laboratório farmacêutico

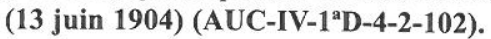


tion pharmaceutique au Portugal. Il y avait une certaine prise de conscience du fait que seules des études semblables à d'autres cursus universitaires permettraient aux pharmaciens portugais d'aspirer à une ascension dans la hiérarchie des professions de santé. Ce n'était qu'avec un support scientifique et technique compatible avec les exigences de la problématique du médicament que le pharmacien portugais pouvait aspirer à une position importante dans la hiérarchie professionnelle. Mais cette question n'a pas été sans conflit au sein de la profession.

\section{Conclusion}

La réforme de l'enseignement pharmaceutique de 1902 a ouvert les portes à une série de réformes de l'enseignement de la pharmacie au Portugal.

La réforme de 1902, bien qu'elle n'ait pas été considérée par les classes dirigeantes pharmaceutiques comme une réforme idéale, fut une réforme jugée réaliste. La nouvelle organisation de l'enseignement pharmaceutique a fortifié l'autorité scientifique et le pouvoir social des pharmaciens portugais.

La réforme de 1902 a introduit un ensemble de savoirs scientifiques et techniques qui rendaient possible une meilleure action au niveau de la santé publique. De nouveaux savoirs ont permis de traiter de manière différente les sujets à aborder, comme, par exemple, l'élargissement du champ d'action des pharmaciens, la dénonciation de l'exercice illégal de la profession et la question de l'exigence de la rigueur scientifique dans la préparation des médicaments.

\section{SOURCES ET BIBLIOGRAPHIE}

\section{Sources manuscrites}

Archives de l'Université de Coimbra, Portugal-AUC

Escola de Pharmacia. Exames de $1^{\mathrm{a}}$ e $2^{\mathrm{a}}$ classes -Livro-AUC-IV-1 ${ }^{\mathrm{a} D} \mathrm{D}-4-2-97$

Escola de Pharmacia. Documentos de matrícula:

1902/03; 1903/04-Livro-AUC-D-CV-4-27 (IV-1 ${ }^{\mathrm{a} D} \mathrm{D}-12-1$ )

1904/05; 1905/06-Livro-AUC-D-CV-4-28 (IV-1 $\left.{ }^{\mathrm{a} D} \mathrm{D}-12-1\right)$

1906/07; 1907/08-Livro-AUC-D-CV-4-29 (IV-1 ${ }^{\text {aDD-12-1) }}$

1908/09; 1909/10-Livro-AUC-D-CV-4-30 (IV-1ªD-12-1)

1910-1911;1911-1912;1912-1913-Livro-AUC-D-CV-4-31 (IV-1ªD-12-1)

Faculté de pharmacie de l'Université de Coimbra

Actas das sessões do Conselho Escolar (1903-1911)

\section{Sources imprimées}

L. Almeida, A Pharmacia. O exercício profissional (a traços rapidos), Coimbra, Imprensa da Universidade, 1904. 

1890

J.S.S. BotTe, Pharmacia. Elementos de pharmacotechnia, Coimbra, Imprensa da Universidade,

J.S.S. BotTE, Pharmacia. Elementos de pharmacotechnia, $2^{\mathrm{e}}$ éd., Coimbra, Imprensa da Universidade, 1899.

E. Collin, Précis de matière médicale, Paris, Octave Doin, 1903.

M.J.F. CoAsta, Hypericum Androscemum, L., Coimbra, Imprensa da Universidade, 1904.

B.G. CraveIro, Algumas considerações sobre a pharmacia portugueza, Coimbra, Imprensa da Universidade, 1903

F. Crollas, B. Moreau, Précis de pharmacie chimique, $2^{\mathrm{e}}$ éd., Lyon, A. Storck \& Cie, 1902.

J.C.R. Dinis, Solanaceas medicamentosas portuguesas. Meimendros (Hyosciamus niger L., Hyosciamus albus L.), Coimbra, Imprensa da Universidade, 1904.

E. DupuY, Cours de pharmacie, 3 vols, Paris, L. Battaille et Cie, 1894.

Fonzes-Diacon, Précis de toxicologie, $2^{\mathrm{e}}$ éd., Paris, A. Maloine, 1912.

V.A. MorA, O chá. Sua composição chimica, preparação e falsificação, Coimbra, Imprensa da Universidade, 1904.

Pharmacopéa Portugueza, Lisboa, Imprensa Nacional, 1876.

L. Prunier, Les Médicaments chimiques, Vol. 1, Paris, Masson et Cie, 1896.

L. Prunier, Les Médicaments chimiques, Vol. 2, Paris, Masson et Cie, 1899.

J.S. Silva, Elementos de analyse chimica qualitativa, Coimbra, Imprensa da Universidade, 1874.

J.S. SILva, Elementos de analyse chimica qualitativa, $2^{\mathrm{e}}$ éd., Coimbra, Imprensa da Universidade, 1883.

J.S. SiLVA, Factoren-tabellen zur ausführung chemischer rechnungen mittels der Von L. Meyer und K. Seubert Gegebenen atomgewichte, Braunschweig, 1887.

J.S. Silva, Elementos de analyse chimica qualitativa, $3^{\mathrm{e}}$ éd., Coimbra, Imprensa da Universidade, 1891.

J.C.A. SoBral, O phosphoro e seus principaes compostos, Coimbra, Imprensa da Universidade, 1904.

J. TARbouriech, Technique des analyses chimiques, 2éd., Paris, A. Maloine, 1906.

\section{BibliograPhIE}

C. Cabral, L. Salgueiro, J.R.Pita, « Le Laboratoire de pharmacognosie de la Faculté de pharmacie de l'Université de Coimbra (Portugal) : enseignement et recherche (1902-1980) », Rev. Hist. Pharm., 2016, n 389, p. 81-92.

J.P.S. DiAs, A Farmácia em Portugal - uma introdução à sua história, 1338-1938, Lisboa, ANF, 1994.

J.C.R. DinıZ, « O ensino farmacêutico na Universidade de Coimbra. Sua evolução desde 1902 até à reforma de 1932 », Notícias Farmacêuticas, Coimbra, 1938, vol. 4, n 3-4, p. 129-204.

J. Esteva De SAgrera, Historia de la Farmacia. Los medicamentos, la riqueza y el bienestar, Barcelona, Masson, 2005.

O. LAFONT, Dictionnaire d'histoire de la pharmacie : Des origines à la fin du XIX $X^{e}$ siècle, $2^{\mathrm{e}}$ éd., Paris, Pharmathèmes, 2007.

J.R. PITA, « Sanitary normalization in Portugal: pharmacies, pharmacopoeias, medicines and pharmaceutical practices (19th-20th Centuries) » in L. ABREU, European Health and Social Welfare Policies, Brno, Brno University of Technology-Vutium Press, 2004, p. 434-453. 

2009.

J.R. PITA, A Escola de Farmácia de Coimbra (1902-1911), Coimbra, Imprensa da Universidade,

J.R. PITA, A.P. Brojo, « Subsídios para a História do ensino farmacêutico na Universidade de Coimbra, no período de1902 a 1988 », Bol. Fac. Farm. Coimbra, 1989, nº 1, p. 7-35.

J.R. PitA, A.L. Pereira, «A Europa científica e a farmácia portuguesa na época contemporânea », Est. Séc. XX, 2002, n 2, p. 231-265.

J.R. PITA, A.L. PEREIRA, « Saberes e micropoderes às portas do séc. XX. Na rota da identidade farmacêutica : o caso coimbrão " in J.R. PITA, A.L. Pereira, Rotas da Natureza. Cientistas, viagens, expedições e instituições, Coimbra, Imprensa da Universidade, 2006, p. 169-174.

M.A. Rodrigues, Memoria Professorum Universitatis Conimbrigensis, Coimbra, Arquivo da Universidade de Coimbra, 1992.

\section{RÉSUMÉ}

L'École de pharmacie de l'Université de Coimbra (1902-1911) - L'étude a porté sur l'École/ Faculté de pharmacie de l'Université de Coimbra (entre 1902 et 1911) sous le régime des études de 1902, en se focalisant sur l'institution, l'enseignement, la recherche réalisée, avec, comme toile de fond, le contexte politique, social et scientifique. Cette réforme ne concernait pas seulement sur le programme des études. C'était aussi une réforme de l'enseignement et une réforme des Écoles de pharmacie qui s'appliqua à l'École de pharmacie de l'Université de Coimbra, mais aussi aux Écoles de pharmacie de Lisbonne et Porto.

\section{SUMMARY}

The Coimbra University Pharmacy School (1902-1911) - The authors study Coimbra University School/Faculty of Pharmacy (between 1902 and 1911) during the 1902 educational reform, centering their attention on the institution, its teachings, the research developed, taking into consideration the political, social and scientific context. This educational reform did not only pertain the study plan. It was also an educational reform and a reform of the Pharmacy Schools, which applied to Coimbra University Pharmacy School as well as to Lisbon and Oporto Pharmacy Schools.

\section{MotS-CLÉS}

Faculté de pharmacie de l'Université de Coimbra, enseignement pharmaceutique, histoire de la pharmacie, Portugal, $\mathrm{XX}^{\mathrm{e}}$ siècle.

[Note : La recherche qui a donné lieu à cette publication s'intègre dans les activités scientifiques du Groupe d'histoire et de sociologie de la science et de la technologie du Centre d'études interdisciplinaires du XXe siècle de l'Université de Coimbra - CEIS20 (UID/HIS/00460/2013) et de la Faculté de pharmacie de l'Université de Coimbra, Portugal.] 